\title{
A Case Study and Meta-Analysis of Type 2 Diabetes Research
}

\author{
Michael L. Gracia \\ Department of Anthropology, University of North Carolina, Chapel Hill, USA \\ Email: mgracia@live.unc.edu \\ Received March $7^{\text {th }}, 2013$; revised April $7^{\text {th }}, 2013$; accepted April 16 $6^{\text {th }}, 2013$
}

\begin{abstract}
Copyright (C) 2013 Michael L. Gracia. This is an open access article distributed under the Creative Commons Attribution License, which permits unrestricted use, distribution, and reproduction in any medium, provided the original work is properly cited.
\end{abstract}

\begin{abstract}
In the last decade, a new wave of inspired research surrounding a transcription factor's role in blood-sugar homeostasis has emerged. Transcription Factor 7-like 2 (TCF7L2 or TCF4), a member of the Wnt signaling pathway, is intimately involved in suppressing glucagon synthesis. It also may affect $\beta$-cell function. Regiospecific trends concerning the selection of its molecular variants, their dependency on environmental and behavioral changes, and their relative associations with type 2 diabetes (T2D) have become well documented. This dynamic and nuanced understanding of one T2D risk factor should be desired in all other phases of study. An open and incorporative mindset such as this can revitalize the research field.

Keywords: Type 2 Diabetes; T2D; Transcription Factor 7-Like 2; TCF7L2; TCF4; West Africa; East Asia; World Health
\end{abstract}

\section{Prologue}

As a traveling physician, you are summoned to West Africa. The secular humanitarian-aid NGO Médecins Sans Frontières has established a proxy in Freetown, Sierra Leone, and Conakry, Guinea adding 800 beds and rehydration points in response to a cholera epidemic catalyzed by the rainy season. Somewhere along the border of Guinea and Sierra Leone, you find a young man suffering from polyuria, blurred vision, fatigue, and other discrete symptoms of type 2 diabetes. Although his long and slender build is revealing enough, you take a crude measure of his body mass index (BMI) to confirm that he is underweight. Genetic predisposition, dietary considerations, and Metformin are all vital components of your prognosis and treatment. Your diagnosis, measurements, and observations combine to reveal a very unique incidence of a metabolic disorder thought by the majority of clinicians to be intimately tied to obesity.

In East Asia, you volunteer to support relief efforts in Indonesia where a 7.6 magnitude earthquake struck the western coast of Sumatra. While supplementing psychiatric support in Padang, you treat a slightly obese, elderly man showing cognitive dysfunction after being brought to the hospital for strokelike symptoms. In this case, your diagnosis of diabetes is much more straightforward, and in your follow-up, you emphasize a dedication to exercise and several lifestyle changes in addition to the supplementary insulin.

\section{Introduction}

All in all, these two hypotheticals reveal the elegance and pathophysiological diversity of one of humanity's most common forms of suffering. Type 2 diabetes affects 285 million people worldwide, a 900 percent increase from a census taken in 1985 (WHO). In this article, we dive deeply into and explore a case example of a specific genetic and micro-regional dimension of type 2 diabetes, emphasizing the environment's role in governing pleiotropic trade-offs in West Africa and East Asia. Transcription factor 7-like 2, a multi-regulatory transcription factor, is a perfect candidate for such an analysis by its dual involvement in blood sugar homeostasis and regiospecificity within those areas. All the while, we synthesize a new argument that combines this information with lessons learned from an analogous, better understood phenomenon that has governed distributions of malaria and sickle cell anemia across Africa for thousands of years. These genetic, micro-regional, and parallel ways of thinking illuminate the necessity of a multiple systems approach to studying type 2 diabetes and reveal a method of bringing focus to the ambiguities and voids still remaining within type 2 diabetes research.

\section{Epidemiological Overview}

Type 2 diabetes (T2D) has become a part of the human condition; it is a global phenomenon with a distribution that reaches across the entire spectrum of human populations. In 1982, P. Zimmet, an Australian-born diabetologist, offered "An Epidemiological Overview of type 2 (non-insulin-dependent) diabetes". Concerning the world distribution, the paper clings to the idea that the recent rise in diabetes incidence mirrors the equally prominent rise in "westernized lifestyles" among new and old populations (Zimmet, 1982). T2D incidence is lowest in isolated populations such as Inuit, Alaskan Athabascan Indians, and scattered East Asian populations still living a "traditional lifestyle" (Zimmet, 1982). Conversely, diabetes prevalence is highest in American Indians, specifically the American Pima Indians; urbanized Pacific Islanders like the Micronesian population of the Nauru; and among migrant South Asian Indian communities within Singapore, Malaysia, and South Africa (Zimmet, 1982). This notion of higher susceptibility among immigrant South Asian Indians resonates well with a growing awareness of a varying degree of "genetic predisposition" for 
T2D existing among all people. Spikes in incidence rates that are aligned with the immigration of certain groups to a new environment suggest a preexisting set of genetic conditions that are maladaptive to that new environment. Finally, the overview concludes with a survey of "universal" environmental and life history factors that, in all likelihood, do indeed contribute to type 2 risk among any given population. These factors include age (specifically the role of diminishing glucose tolerance associated with the aging process), obesity (a metric that the World Health Organization (WHO) expert committee concludes to be the most powerful predictor of T2D incidence), physical activity, dietary factors, and parity (Zimmet, 1982). Despite the age and usage of archaic terminology for describing certain populations in Zimmet's article, there are many lessons that can be learned from it concerning research fallacies therein-many of which are still employed today.

Two questions immediately come to mind with this sort of analysis concerning the varying genetic susceptibility of different "ethnic groups". Firstly, who are these ethnic groups? Native American Indians throughout the Americas have tended to lose their genetic isolation and recognizability post-colonization. Therefore, any claim reliant on these sweeping notions of genetic lineages and predispositions becomes almost meaningless. We must be sensitive and aware of the persistence of the mosaic of evolutionary forces and gene flow between human populations that these "macro ethnic groups" cannot encapsulate. Secondly, for what is the term "genetic predisposition" actually being used in descriptions such as these? The networks of interactions of the human genome with itself, its environment, and even the genomes of other organisms is so complex and daunting that it is unclear whether implementation of such a loaded term like "genetic predisposition" is being used to signify a new research avenue or a dead end. This sort of terminology has been used as a sort of umbrella term-a catch all - that can be negligent to the phenomena at hand and a deterrent to deeper research. Despite this fact, many researchers have been bold enough to enhance our focus and illuminate the interconnectedness between the microbiological, genetic, and regional factors of type 2 diabetes in a far less reductive manner. These studies, emphasizing the subtleties of the condition, paint type 2 diabetes in a brand new light and can help usher in a new era of productivity within diabetes research.

\section{Subtleties}

The mezcla or mixing of risk factors and predispositions surrounding type 2 diabetes (T2D) can be overwhelming. In order to demystify and partially deconstruct this ever-complex disorder, we can view T2D with respect to a single protein. Transcription factor 7-like 2 (TCF7L2) is a pleiotropic gene that, among its many roles in regulation, serves as a vital member of the Wnt signaling pathway. By associating with CTNNB1 (previously $\beta$-catenin) within the cell nucleus, TCF7L2 acts on Wnt target genes that inhibit the production of a glucagon precursor in enteroendocrine cells of the pancreas (Smith, 2006). Within the liver, glucagon activates the breakdown of glycogen into glucose and its release into the bloodstream, therefore serving to increase blood sugar and directly antagonize insulin. With this role in blood sugar homeostasis, it is easy to appreciate the subtle, yet powerful physiological relation existing between TCF7L2 and type 2 diabetes.

However, in order to increase the depth and breadth of our analysis of the relationship between TCF7L2 and type 2 diabetes, we can also consider the gene that encodes it. By viewing a discrete gene involved in $\mathrm{T} 2 \mathrm{D}$, we are able to compare demographic and cross-species data to uncover evolutionary, life history, and regional trends at the most fundamental level of human genetics and evolutionary biology. Transcription factor 7-like 2 gene (TCF7L2 gene), also called TCF4 gene, constitutes a 216 kilobase $(\mathrm{kb})$ region on chromosome 10 within the human genome. PhastCons and PhyloP software techniques have been used to analyze conservation across a variety of vertebrates, revealing tracks of alignment among 44 species. These software tools, under the "PHAST" package, are capable of comparing across cell lines to in order to indicate directional selection when allelic frequencies are changing at rates that cannot be accounted for by genetic drift alone. They can also reveal patterns of conservation and decelerated evolution, if changes in frequency fall below simulations that assume stable equilibrium with respect to those alleles. Furthermore, they can compare the given likelihood of each individual nucleotide within the sequence belonging to the "conserved element" by analyzing the individual alignment column relative to its flanking columns. Among the vertebrates found to posses a conserved sequence in the coding region were placental mammals such as mice, bats, and, most relevant to human evolution, chimpanzees (Pan troglodytes) and gorillas (UCSC Encyclopedia of DNA Elements). Visualizations of the alignment among placental mammals and other select vertebrates may be found in Figure 1. Notice the increasing overlay as the Multiz alignment transcends from the Stickleback and other out-groups to the Rhesus monkey. The high degree of overlap within the PhyloP analysis, however, suggests that the region is highly conserved among placental mammals.

Several mutations of TCF7L2 gene have been implicated for heightened type 2 risk. Among them is an array of single nucleotide polymorphisms (SNPs) - single base pair substitutions created along the coding sequence. Two SNPs in particular, markers rs12255372 and rs7903146, have been gaining momentum among researchers in reference to their connection to T2D. Munoz et al. have linked presence of the marker rs12255372 to a reduction in insulin secretion among non-diabetic women of African and European descent - a subtle and distinct effect not attributable to the Wnt pathway. The pathway (in which TCF7L2 has been introduced as a vital member) has a paramount role in glycolysis and therefore in positive increases of blood sugar, but says nothing about insulin, the blood peptide governing sugar uptake by cells. These researchers used multiple clinical and metabolic parameters, such as BMI, percent body fat, insulin sensitivity $(\mathrm{Si})$, acute insulin response to glucose (AIRg), and the disposition index (DI) to confirm an association between homozygosity for the at-risk allele (TT) and alteration of these metabolic characteristics in ways that contribute to one's risk. (Munoz, 2006). Munoz et al. further propose a mechanism behind this increased risk, positing that TCF7L2 directly affects $\beta$-cell function. More specifically, they suggest that "TCF7L2 influences insulin secretion and may affect susceptibility for type 2 diabetes by modulating the adequacy of insulin secretion to compensate for the prevailing degree of insulin resistance" (Munoz, 2006). This hypothesis introduces a brand new role of the transcription factor in both senescence and in stimulating insulin secretion within $\beta$-cells in response to increasing insulin resistance and could 


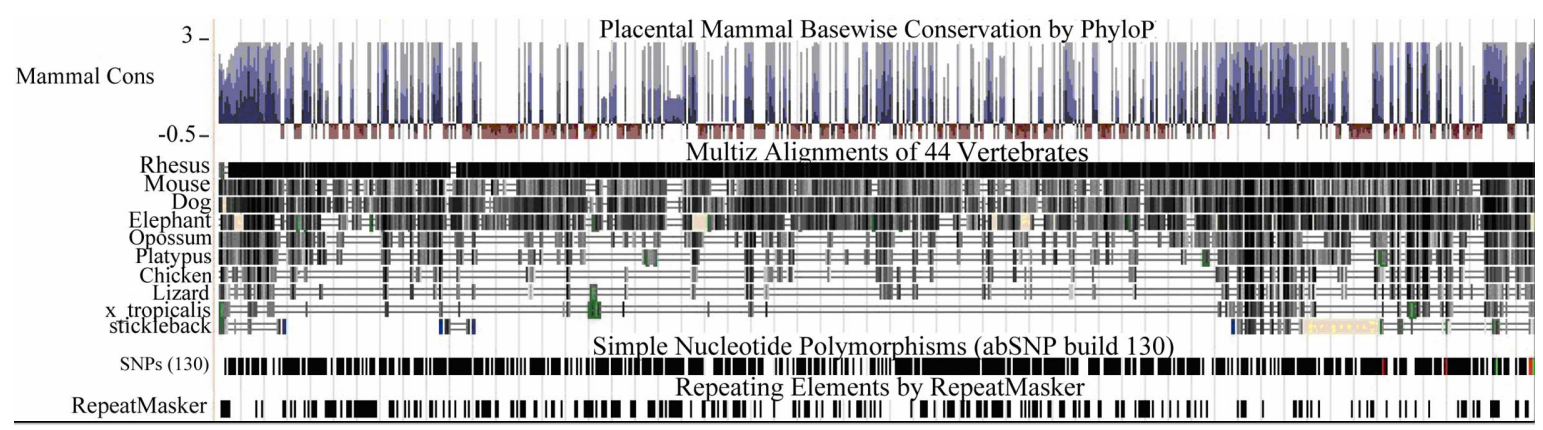

Figure 1.

Mulitz alignment and PhyloP analysis.

explain the heighted risk of T2D among those expressing rs 12255372 if the mutation is, in fact, deleterious with respect to that function.

Furthermore, another thread of research has emerged out of Iceland and Germany in 2006 concerning the association between rs7903146 and heightened risk of type 2 diabetes and various forms of colorectal cancer (Grant et al., 2006). These endeavors question the regiospecificity of T2D by sampling from various populations worldwide. One particular letter to Nature: Genetics, "Refining the impact of TCF7L2 gene variants on type 2 diabetes and adaptive evolution", further defines the risk association between TCF7L2 and diabetes among a selected sample of West Africans by hypothesizing its connection to a particular variant, $\mathrm{HapB}_{\mathrm{T} 2 \mathrm{D}}-\mathrm{a}$ molecular variant produced by homozygosity for rs7903146. Researchers introduce rs7903146 not as a recent polymorphism in the evolutionary history of the sequence, but as an "ancestral" allele to the current wild type (Helgason et al., 2007). Data further supporting the ancestral allele's association to T2D were replicated in roughly 1000 Moroccans in a secondary meta-analysis conducted by Cauchi et al. (2007). Research also suggests that a second, less understood molecular variant of TCF7L2, HapA, can also indicate type 2 risk and is, in fact, experiencing positive selection in some agrarian communities in West Africa, Europe, and East Asia by its association with body mass index (BMI) (Helgason et al., 2007).

To prove these hypotheses, Helgason et al. (2007) genotyped a group of 1149 affected Danish individuals among 2400 controls and 621 affected West Africans among 448 controls. These demographic groups were chosen because of the high incidence rates among them. After the second wave of genotyping, it was discovered that one of the markers, with respect to relative risk, stood out among the rest. The SNP rs7903146 yielded a relative risk $(\mathrm{RR})=1.49$ with a $95 \%$ confidence interval among the Danish group, and a RR $=1.45$ in West Africa, values much higher than those produced by the other haplotype (rs12255372). Helgason et al. (2007) also inquire about the possible positive selection for HapA, another variant of TCF7L2 occurring within certain West African, European, and East Asian populations, on the grounds that this variant is associated with high BMI and alters concentration of hunger-satiety hormones that positively affect energy metabolism in more energy-plentiful environments. Indeed, molecular-clock dating analyses align the emergence of HapA with the transition to agriculture within each HapMap group (Helgason et al., 2007). In a second assay, they performed both a Fixation Index (FST) test and long-range haplotype (LRH) test within these populations in the hopes of finding evidence supporting positive selection. Realized fixation index values $(\mathrm{FST}=.306)$ suggested that this sort of genetic differentiation is unlikely via neutral evolution. Additionally, high LRH results, a statistic that gauges the relative haplotype homozygosity within a population, further support the presence of positive selection. High FST values and high homozygosity suggest a rapid rate of divergence where neutral, background mutation is insignificant. SNP rs7903146 and variant HapA are both relevant risk factors to type 2 diabetes within their respective populations, though their physiological connections to the disease may differ significantly (Helgason et al., 2007).

This article illuminates some of the subtle complexities of natural selection. It is important to remember that natural selection does not work to create a super organism or to rid an organism of all impurities. In fact, evolution and natural selection walk a very fine line. These processes work to strike a perfect note in resource allocation due to the pleiotropic reality of life on earth, a constant re-equilibration, at the genetic level, between relative benefit and disadvantage. There is no free lunch in evolution. This is a fact highlighted in the positive selection for HapA. Although it has been illustrated as a risk factor for diabetes, authors speculate that HapA's reproductive success despite this fact is due to its advantageous effects on BMI relative to energy metabolism in agricultural environments (Helgason et al., 2007). With regards to human disease in general, we can take some additional time to ponder and appreciate the ways in which different physiological pathways can arrive at a single disorder. HapA has been strongly correlated with high BMI increases among certain groups of West Africans, Europeans, and East Asians in more energy-plentiful environments, while the haplotypes rs12255372 and rs7903146 (HapB) have been found unassociated, and at times negatively associated, with BMI among other sampled populations of North and West Africa. Instead, the latter variant contributes to T2D risk by affecting insulin secretion in the ways previously described (Helgason et al., 2007). These researchers were respectful and thoroughly sensitive to the genetic nature and variability of their subject matter. Their attention to regiospecificity brings a new, important perspective to understanding these risk factors, because any discussion of selective pressures or human evolution cannot exist without intense consideration for the environments in which populations exist. Both the methodology executed and conclusions posited in Helgason et al. (2007) put forth creative techniques and viewpoints in studying $\mathrm{T} 2 \mathrm{D}$, in turn adding another wrinkle of understanding to the disorder's complex epidemiology. 


\section{An Analogy}

A metaphor exists comparing the relationship between body mass index and type 2 diabetes in Africa and East Asia to the rise and distribution of malaria and sickle cell trait across the African continent. Both series of evolutionary phenomena revolve around the implementation of agriculture and the subsequent rise in human population size and societal complexity. Retro-analysis and comparison of the co-evolution between $P$. falciparum and humans to the genetic changes that positively affect BMI despite T2D risk in some African populations can further reframe diabetes in our minds as a dynamic and subtle consequence of human behavioral history. This analogy is meant to remind us of the environment's powerful effect on the genome, to add context and understanding to the trends we have studied thus far, and to affirm a novel perspective for studying T2D and other emergent disorders in the future.

To learn from this analogy, one needs to retrace the concurrent adaptation of a protozoan with macro-scale behavioral changes of the human species. The "burden" of malaria, as described in Richard Carter and Kamini Mendis' survey of the mosquito-borne infectious disease, has been felt by invertebrates, vertebrates, mammals, and primates throughout evolutionary history. Plasmodium falciparum, the most virulent human malaria agent, may have diverged from Plasmodium reichenowi in concert with the split between the precursors of humans and chimpanzees around 4 to 10 million years ago (Carter \& Mendis, 2002). P. falciparum and other extant Plasmodium agents of human malaria- $P$. malariae, $P$. ovale, and $P$. vivax - are also equally tied to the evolution of their vector, the female Anopheles mosquito. These realities, perhaps better than any other, truly embody the intimate coevolutionary relationship existing between the extant Plasmodium agents of human malaria and their hosts.

Eight to ten thousand years ago in the "Fertile Crescent" of modern day southern Turkey and northeastern Iraq, modern humans began changing their behavior, and their surroundings, in a momentous way. The innovation of agriculture - a methodological practice involving the clearing, irrigating, cultivating and harvesting of fertile land-would allow humans an unprecedented level of sustainability and control over their environment. This newfound control would direct the species' global distribution and evolution, as well as those of the species dependent on them. Four to five thousand years ago, the agrarian revolution reached western and central Africa. Human populations across Africa began gathering in larger settlements and reproducing at much higher rates, now supported by this new form of energy production (Carter \& Mendis, 2002).

The behavioral adaptations of humans caused the African Anopheles to follow in suit. The anthropophilic index, the probability that a mosquito in a given region will acquire a blood meal from a human source, is a good proxy for gauging this coevolution. Carter and Mendis cite anthropophilic indices taken from various regions of the world in the range of $10 \%$ $50 \%$. In sub-Saharan Africa, however, the range of indices is $80 \%-100 \%$ (Carter \& Mendis, 2002). Frank Livingstone was the first to attribute this stark shift in preference to the significant changes in human density and population size created by the implementation of agriculture. It has been hypothesized that the subtropical, cultivated regions of Asia and the Middle East were shielded from this trend by presenting mosquitos with a larger buffer of domesticated animals, thereby dampening their dependency on human activity and distribution. Furthermore, in Africa, the practice of agriculture also created large collections of still water that facilitated mosquito breeding. The increases in human density and local population size along with the addition of mosquito habitats near these population centers combined to create a situation of endemic malaria in certain regions of Africa (Carter \& Mendis, 2002). From an ecological perspective, the invention of agriculture can and should be viewed as a major disturbance within the community. Furthermore, human beings can be viewed as ecological engineers within their ecosystem. By manipulating their environment, they also facilitated the re-colonization and proliferation of another dependent species within the successional process.

We must now track a final aspect to this story-the frequencies and selection for human HgbS, the allele causing the autosomal recessive genetic blood disorder sickle-cell anemia. While homozygosity for this allele produces the commonly mortal phenotype we call sickle-cell anemia, a heterozygous phenotype produces a co-dominant effect we call "sickle-cell trait". Those bearing the heterozygous phenotype produce a blend of normal red blood cells and "sickled" cells_-abnormal red blood cells that acquire their distinct shape from a deformity in the $\beta$-globin chains of hemoglobin. It is the presence of these sickled cells among functional red blood cells that offers carriers both adequate perfusion of body tissue and an intraerythrocytic environment that inhibits $P$. falciparum's lifecycle and proliferation. Increases in post-infection survivability among carriers, estimated to be as high as $90 \%$ in some regions, have created intense selective pressure for this heterozygous advantage in regions of endemic malaria (Carter \& Mendis, 2002).

To learn and apply the lessons concerning agriculture, malaria, and sicke-cell trait to selective forces in agrarian societies that affect the distribution of type 2 diabetes, we must think laterally. While agriculture as a common denominator makes this an easy comparison, direct similarities in this meta-analysis are not as important as the underlying principles they imply. Agriculture and its effects on Plasmodium, Anopheles, and humans serve as an example of behavioral and environmental catalysis of adaptive evolutionary change. In the same way that the practice of agriculture has created significant selective pressures that have increased the frequency of the $\mathrm{HgbS}$ allele despite the devastating effects of the recessive homozygous genotype, agriculture has created an energy abundant environment where a molecular variant of TCF7L2 (HapA), despite its positive association with BMI and type 2 diabetes, can thrive because of its advantages in energy metabolism. Additionally, while we have learned a substantial amount from the similarities of these examples, we can also learn from the subtle ways in which they differ. It is important to distinguish that while causality of these evolutionary relationships are similar, selection for HapA is not being driven by the environmental side effects of agriculture, but rather by the production of agriculture itself. The various new forms and quantities of food possible in agricultural societies have forever changed the dietary patterns within them. This different form of behavioral change has shown equally capable of influencing genetic change, and the persistence of type 2 diabetes. One must remember that diet, along with subsistence, infectious disease, and other sociocultural and environmental filters must be given the attention they deserve in the study of any worldly distributed disorder (Jackson, 2004). Both these filters - by their ability to fill the gap and complicate the pathway between a coded genotype and 
an expressed phenotype - and the regional subtleties already described have a lot to tell us if we are willing to listen.

\section{Conclusion}

A meta-analysis superimposed on this case study has been meant to promote the consideration of subtle, often pleiotropic genetic relationships, micro-regional analysis, population substructure, and the role of the environment over notions of macro-ethnic groups and uninspired dogmas revolving around "westernization" as a universal cause. Often within the literature, type 2 diabetes is treated as a static and isolated condition, and studies on its behalf have been plagued by these ambiguous claims along with narrow-focused, absolutist parameters that obsess with obesity and insulin secretion. Together, these two foci in research have combined to create a mindset that ignores many of the subtitles within the condition and its ties to other facets of life and history.

To bring a new mindset to life, we were introduced to TCF7L2, a transcription factor coded in a highly conserved sequence of the genome and intimately involved in glycolysis and $\beta$-cell function. Several single nucleotide polymorphisms (SNPs) of TCF7L2 gene have been positively associated with type 2 diabetes. The molecular variant that arises from these polymorphisms $\left(\mathrm{HapB}_{\mathrm{T} 2 \mathrm{D}}\right)$ and another molecular variant that is less understood (HapA) each increase risk for T2D by either directly affecting $\beta$-cell function within the pancreas or by positively associating with BMI. The persistent, and in some cases, growing frequencies of these variants in studied populations is often the result of balancing selection dictated by the pleiotropic reality of TCF7L2 gene and changes in human environments and behavior. One such example of this pleiotropic tradeoff is the selection of HapA in some African populations. As an adaptive mechanism, HapA is still being selected for in certain agricultural environments despite its positive association with type 2 risk because of the advantages in energy storage and metabolism of higher BMI. This causal relationship is similar in principle to the ways in which agriculture has governed the frequencies and distribution of malaria and sickle cell trait across the African continent.
Just as humans continue to adapt and evolve, so too must the body of literature surrounding type 2 diabetes. The literature is a reflection of our mindset, and our mindset needs to change. These realizations allow us to reframe diabetes as a highly delicate and variable human condition, and to place it within a matrix of adaptive evolution, the environment, human behavior, and human history.

\section{REFERENCES}

Carter, R., \& Mendis, K. N. (2002). Evolutionary and historical aspects of the burden of malaria. Clinical Microbiology Reviews, 15, 564594. doi:10.1128/CMR.15.4.564-594.2002

Cauchi, S. et al. (2007). TCF7L2 is reproducibly associated with type 2 diabetes in various ethnic groups: A global meta-analysis. Journal of Molecular Medicine, 85, 777-782. doi:10.1007/s00109-007-0203-4

Encyclopedia of DNA elements. Santa Cruz: University of California. URL (Last checked 19 April 2012).

$<$ http://genome.ucsc.edu/ENCODE/>

Grant, S. F. et al. (2006). Variant of transcription factor 7-like 2 (TCF7L2) gene confers risk of type 2 diabetes. Nature Genetics, 38, 320-323.

Helgason, A. et al. (2007). Refining the impact of TCF7L2 gene variants on type 2 diabetes and adaptive evolution. Nature Genetics, 39, 218-225. doi: $10.1038 / \mathrm{ng} 1960$

Jackson, F. L. C. (2004). Human genetic variation and health: New assessment approaches based on ethnographic layering. British Medical Bulletin, 69, 215-235. doi:10.1093/bmb/ldh012

Livingstone, F. (1958). Anthropological implications of sickle cell gene distribution in West Africa. American Anthropologist, 60, 533-562. doi:10.1525/aa.1958.60.3.02a00110

Melzer, D. et al. (2006). Effects of the diabetes linked TCF7L2 polymorphism in a representative older population. BMC Medicine, 4, 34.

Munoz, J. et al. (2006). Polymorphism in the transcription factor 7-like 2 (TCF7L2) gene is associated with reduced insulin secretion in nondiabetic women. Diabetes, 55, 3630-3634. doi: $10.2337 / \mathrm{db} 06-0574$

Smith, U. (2007). TCF7L2 and type 2 diabetes-We WNT to know. Diabetologia, 50, 5-7. doi:10.1007/s00125-006-0521-z

World Health Organization. Fact sheet N ${ }^{\circ} 312$. URL (Last checked 19 April 2012).

$<$ http://www.who.int/mediacentre/factsheets/fs312/en/> 